\title{
Differential expression and inhibitory effects of aquaporins on the development of adenomyosis
}

\author{
LIPING JIA, YUZHU LIU, YIXU HAN, XIAOFEI ZHOU and FAHUI WANG \\ Department of Gynecology, The Second Affiliated Hospital of Hainan Medical University, \\ Haikou, Hainan 570311, P.R. China
}

Received November 15, 2019; Accepted June 4, 2020

DOI: $10.3892 / \mathrm{mmr} .2020 .11479$

\begin{abstract}
Aquaporin 2 (AQP2), AQP5 and AQP8 participate in adenomyosis (AM). However, the roles of these three molecules in AM have not been fully elucidated. In the present study, Institute of Cancer Research female mice were used to establish a model of AM. Subsequently, the endometrial tissues of the mice were observed by hematoxylin-eosin staining, and AM severity, uterus diameter, uterus index, ovary index and numbers of nodules on the uterine surface were evaluated and counted. In addition, eutopic and ectopic endometrial stromal cells (ESCs) were isolated from eutopic and ectopic endometrial samples derived from patients with AM and were then identified by immunofluorescence. The viability, and migratory and invasive ability of ESCs transfected with small interfering RNA targeting AQP5 (siAQP5) were determined by Cell Counting Kit-8, scratch wound-healing and Transwell assays, respectively. Reverse transcription-quantitative polymerase chain reaction was performed to determine the mRNA expression levels of AQP5, epithelial-mesenchymal transition (EMT)-related genes (E-and N-cadherin), matrix metalloproteinase (MMP)-2 and -9. Protein expression levels of AQP2, AQP5, AQP8, E-, N-cadherin, MMP-2 and -9 were detected by western blotting. AM severity and uterus index were higher, and there were a greater number of nodules on the uterine surface in the AM group compared with the sham group. $\mathrm{AQP} 2, \mathrm{AQP} 5$ and AQP8 proteins were highly expressed in eutopic and ectopic endometrium of the AM group, and AQP5 was more highly expressed than AQP2 or AQP8. In addition,
\end{abstract}

Correspondence to: Dr Liping Jia, Department of Gynecology, The Second Affiliated Hospital of Hainan Medical University, 48 Baishuitang Road, Haikou, Hainan 570311, P.R. China

E-mail: jialip_ping@163.com

Abbreviations: AM, adenomyosis; AQP2, aquaporin 2; ESCs, endometrial stromal cells; MMP, matrix metalloproteinase; EMT, epithelial-mesenchymal transition; AQPs, aquaporins; ICR, Institute of Cancer Research; HE, hematoxylin-eosin

Key words: matrix metalloproteinase-2, -9, migration, adenomyosis, aquaporin, invasion, epithelial-mesenchymal transition the data showed that Vimentin was positively expressed in ESCs, and that siAQP5 suppressed the mRNA expression levels of AQP5, cell viability, migration, invasion, EMT and MMP-2 and -9 expression in ESCs. In conclusion, AQP2, AQP5 and AQP8 were highly expressed in eutopic and ectopic endometrium. Notably, AQP5 silencing may suppress AM by inhibiting viability, migration, invasion, EMT, and MMP-2 and -9 expression in ESCs.

\section{Introduction}

Adenomyosis (AM) is a heterogenous gynecological disease, which is characterized by uterine enlargement, and deep location of endometrial glands and stroma within the myometrium (1). Menorrhagia, dysmenorrhea and metrorrhagia are the most frequently identified symptoms in two thirds of patients with AM; notably, one third of patients with AM are asymptomatic (2). A previous report revealed that preterm birth and premature rupture of membranes, uterine atony and ectopic pregnancy are also associated with AM (3). However, the exact pathogenesis and etiology of AM have not been fully elucidated, due to its complex pathogenesis and variable presentation. Hysterectomy is a standard treatment for AM, and suppressive hormonal treatments cam be used to temporarily suppress AM and improve the symptoms; however, there is no currently available medical therapy to cure AM (4). Therefore, exploring the pathogenesis of AM is critical for developing strategies for treating the disease.

Aquaporins (AQPs) are a family of membrane water channels, which are distributed widely in specific cell types in different organs and tissues, and primarily function as regulators of intracellular and intercellular water flow (5). A previous study identified the significance of AQPs in mammalian pathophysiology, and suggested that AQPs and AQP modulators (mostly inhibitors) could act as underlying drug targets (6). In addition to the research achievements in drug-like AQP inhibitors, regulation of AQP function may be desirable in some pathophysiological conditions, such as in tumors (7). AM is characterized by the malignant biological behaviors of invasion and migration (8); these biological behaviors are similar to those of tumors. Therefore, AQP regulation may be a potential therapeutic option for treating AM. Additionally, AM and endometriosis are closely linked diseases, although there are several differences in 
their pathogenesis and pathogenic mediators (9). Some similarities exist between AM and endometriosis at least in some subgroups, more often the two conditions coexist (10). AQP2, AQP5 and AQP8 have all been reported to play roles in endometriosis (11), but their roles in the occurrence of AM have not been clearly reported. Notably, AQP5 is known to play a role in estrogen-induced ectopic implantation of endometrial stromal cells (ESCs) in endometriosis (12). Furthermore, AQP5 gene silencing has been shown to inhibit the proliferation and migration of ectopic endometrial glandular epithelial cells in endometriosis (13). Therefore, it is of great value to explore the role of AQP2, AQP5 and AQP8 in the progression of AM; such an exploration based on the regulation of AQP2, AQP5 and AQP8 might contribute to the discovery of a novel therapy for AM. To the best of our knowledge, no currently used drug specifically targets AQP5.

In the present study, the expression levels of AQP2, AQP5 and AQP8 were detected in eutopic and ectopic endometrial samples from mice with AM, and the effects of AQP5 silencing on cell viability, migration, invasion, epithelial-mesenchymal transition (EMT), and matrix metalloproteinase (MMP)-2 and MMP-9 expression were detected in eutopic and ectopic ESCs.

\section{Materials and methods}

Animals. Sexually mature virgin female Institute of Cancer Research (ICR) mice (age, 8 weeks) and 8-week-old male ICR mice (Chengdu Dossy Experimental Animals Co., Ltd.) were used in the present study. The mice weighed $\sim 29 \mathrm{~g}$ were freely fed a standard rodent diet from Animal Laboratory of Chengdu University of Traditional Chinese Medicine. The mice were maintained under specific pathogen-free laboratory conditions at $20^{\circ} \mathrm{C}$ under a 12 -h light/dark cycle with $48 \%$ humidity. The animal health and behavior were monitored every 3 days. All animal experiments were approved by the Institutional Committee for Animal Research of The Second Affiliated Hospital of Hainan Medical University (Haiku, China) and in accordance with National Guidelines for the Care and Use of Laboratory Animals (14).

Animal model of AM and collection of specimens. The mice were divided into control (without any surgery), sham (the needle was punctured into the right-side of the uterus and saline solution was injected into the uterine cavity), AM-I (estrus) and AM-II (non-estrus) groups ( $\mathrm{n}=12 \mathrm{mice} /$ group). Mice in the AM-I and AM-II groups received surgery for modeling AM. All groups of mice were fed for 3 months to the end point of the experiment.

The AM animal model was constructed by the transplantation of the pituitary gland of mice into the uterus of female mice, as previously reported $(15,16)$. Briefly, 8 -week old female mice were administered with an appropriate amount of anesthetic (3\% pentobarbital sodium, 30-35 mg/kg) through intraperitoneal injection. The skin layer and subcutaneous muscle layer were stratified in the 8-week-old female mice, and uterine bifurcation under the bladder was used to isolate the uterus. Male mice (age, 8 weeks) were sacrificed by overdose with $100-150 \mathrm{mg} / \mathrm{kg}$ pentobarbital sodium through intraperitoneal injection, followed by cervical dislocation used as a secondary physical method of sacrifice; euthanasia was verified by checking the heartbeat of the mice. Subsequently, craniotomy was performed to extract the pituitary glands from the mice, and the extractions were mixed with saline solution. Part of the pituitary glands was maintained in saline solution suspension $(0.5-1 \mathrm{ml})$ in a needle. The needle was injected into the right-side of the uterus of female mice, and the pituitary-saline suspension was slowly pushed into the uterine cavity. The isolated uterus was put back in the abdominal cavity while the needle and the inner core were removed. A total of 3 months later, all mice were sacrificed (via cervical dislocation) at AM-I or AM-II according to vaginal secretion smears, and the right uterine specimen was immediately collected by laparotomy. Nodule numbers on the uterine surface of mice was counted under a light microscope. The uterine diameter of mice, and uterine, ovarian and body weight were measured by a Vernier caliper (segmented measurement) and electronic balance. Half of uterine tissues were added into Eppendorf tubes and stored in liquid nitrogen, whereas the other uterine tissues of mice were stored in $4 \%$ paraformaldehyde at $4^{\circ} \mathrm{C}$ for protein detection using western blotting. Uterine index (\%) was calculated as follows: Uterine weight $(\mathrm{mg}) /$ mouse body weight (mg) x100. Ovary index (\%) was calculated as follows: Ovary weight (mg)/mouse body weight (mg) x100.

Hematoxylin-eosin (HE) staining and grading of AM severity. Uterine tissues of mice fixed with $4 \%$ paraformaldehyde for $24 \mathrm{~h}$ at room temperature were embedded in paraffin and cut into $4-\mu \mathrm{m}$ sections, which were then stained with $\mathrm{HE}$ for $5 \mathrm{~min}$ at $60^{\circ} \mathrm{C}$. After staining, the sections were dehydrated with gradually increasing concentrations of ethanol and xylene.

Longitudinal and cross sections of uterine tissues of the mice were selected. Under an optical microscope, the areas of eutopic and ectopic endometrium were observed under magnification, x10 and the grade of AM severity of endometrial glands and interstitial permeability of the uterine muscle were observed under magnification, $\mathrm{x} 20$.

The specific grading indicators of AM severity were as follows: Grade 0 (normal uterus), grade 1 (ESCs invaded the inner layer of the muscles), grade 2 (endometrial stroma and gland invaded the inner layer of muscles), grade 3 (endometrial stroma and gland invaded the junction of the internal and external muscle layer) and grade 4 (endometrial gland exhibited cystic hyperplasia and nodules appeared under the serous layer). In each case, the cross section area of the inner membrane was determined by calipers, and AM severity was evaluated according to the degree of permeability (grade 0-4), the average value was calculated to assess the severity of the uterine gland. The experiment was conducted at least in triplicate.

Cells isolation, culture and identification. Six cases of AM (age, 33-51 years) were confirmed by pathology after total hysterectomy at the Second Affiliated Hospital of Hainan Medical University (Hainan, China) between January 16, 2018 and March 16, 2019. The present study was approved by the Ethics Committee of the Second Affiliated Hospital of Hainan Medical University (SA20180111022). Written informed consent was obtained from all patients.

Under aseptic conditions, eutopic and ectopic endometrial samples were collected in a sterile cell culture dish, and 
were then washed 2-3 times with D-Hanks (Beijing Solarbio Science \& Technology Co., Ltd.) to remove the mucus and blood clots attached to the surface of the tissue specimens. The tissue samples were rinsed in sterile PBS and then sliced into small pieces (4-5 $\mu \mathrm{m})$. Subsequently, the sliced tissues were maintained in $50-\mathrm{ml}$ centrifuge tubes, centrifuged at $4^{\circ} \mathrm{C}, 500 \mathrm{x}$ g for $5 \mathrm{~min}$ and the liquid supernatant was removed. Collagenase I (Invitrogen; Thermo Fisher Scientific, Inc.) was added to eutopic or ectopic samples until the volume was raised by 1 or $3 \mathrm{~cm}$, respectively. The eutopic or ectopic endometrial suspension was digested with Collagenase I for 1 or $2 \mathrm{~h}$ respectively in a water bath at $37^{\circ} \mathrm{C}$. In order to promote the effect of Collagenase I, the mixed suspension was shaken every $15 \mathrm{~min}$. Subsequently, DMEM (Hyclone; GE Healthcare Life Sciences) supplemented with $10 \%$ fetal bovine serum (FBS; Gibco; Thermo Fisher Scientific, Inc.) was added into the mixed suspension at an equal volume to Collagenase I to terminate the digestion. The suspension was filtered through a $40-\mu \mathrm{m}$ cell strainer into a fresh $50-\mathrm{ml}$ centrifuge tube. The filtered suspension was centrifuged at $4^{\circ} \mathrm{C}, 800 \mathrm{x}$ g for $5 \mathrm{~min}$, and the liquid supernatant was discarded. Precipitates (cells) in the tube were resuspended in DMEM supplemented with 10\% FBS. The cells were plated into a $25-\mathrm{cm}^{2}$ culture flask at density of $1 \times 10^{5}$ cells $/ \mathrm{ml}$ and cultured at $37^{\circ} \mathrm{C}$ with $5 \% \mathrm{CO}_{2}$.

After the ESCs were cultured to generation 2-3, the expression levels of Vimentin were detected by immunofluorescence with an anti-Vimentin antibody (cat. no. PA5-27231; Invitrogen; Thermo Fisher Scientific, Inc.).

Immunofluorescence staining. ESCs were cultured on $1 \times 1 \mathrm{~cm}$ cover slips, fixed with $4 \%$ cold polyoxymethylene (cat. no. P1110; Beijing Solarbio Science \& Technology Co., Ltd.) for $20 \mathrm{~min}$ at $4^{\circ} \mathrm{C}$ and then washed three times with PBS. The fixed cells were subsequently incubated in PBS containing $0.2 \%$ Triton X-100 for 2-5 min and then blocked with 5\% BSA (Sigma-Aldrich; Merck KGaA) for $1 \mathrm{~h}$ at room temperature. Next, the cells were incubated with an anti-Vimentin antibody (1:100; cat. no. PA5-27231; Invitrogen; Thermo Fisher Scientific, Inc.) overnight at $4^{\circ} \mathrm{C}$, washed and incubated with biotin-conjugated anti-rabbit IgG (1:8,000; cat. no. 65-6140; Invitrogen; Thermo Fisher Scientific, Inc.) at $37^{\circ} \mathrm{C}$ for $1 \mathrm{~h}$. The nuclei were stained with 4',6-diamidino-2-phenylindole (DAPI; cat. no. D9542; Sigma-Aldrich; Merck KGaA) for $10 \mathrm{~min}$ at room temperature in the dark. Fluorescence images were captured using an immunofluorescence laser confocal microscope (Nikon Corporation). The experiment was performed at least in triplicate.

Transfection. Parental non-transfected cells were used as control cells. The small interfering RNA (siRNA) targeting AQP5 (siAQP5, 5'-GCGCUCAACAACAACACAA-3') and scrambled/nonsense siRNA negative control (siNC, 5'-ACG UGACACGUUCGGAGAATT-3') were purchased from Guangzhou RiboBio Co., Ltd. The cells transfected with siNC served as the negative control for siAQP5. The siRNAs $(0.25 \mu \mathrm{g})$ were transfected into the eutopic and ectopic ESCs $\left(5 \times 10^{5}\right)$ at $37^{\circ} \mathrm{C}$ for $48 \mathrm{~h}$ to knockdown AQP5 expression using Lipofectamine ${ }^{\circledR} 2000$ Transfection Reagent (cat. no. 11668; Invitrogen; Thermo Fisher Scientific, Inc.).
Table I. Primer sequences used for reverse transcriptionquantitative polymerase chain reaction.

\begin{tabular}{ll}
\hline Primer & \multicolumn{1}{c}{ Primer sequences (5'-3') } \\
\hline AQP5 F & CAAAGACAGCGGCAGAGACTAT \\
AQP5 R & CTGTCTCCTTTTCCAGGTTATCC \\
MMP-2 F & TGGCAAGTACGGCTTCTGTC \\
MMP-2 R & TTCTTGTCGCGGTCGTAGTC \\
MMP-9 F & GCTGACTACGATAAGGACGGCA \\
MMP-9 R & TAGTGGTGCAGGCAGAGTAGGA \\
E-cadherin F & CGAGAGCTACACGTTCACGG \\
E-cadherin R & GGGTGTCGAGGGAAAAATAGG \\
N-cadherin F & TCAGGCGTCTGTAGAGGCTT \\
N-cadherin R & ATGCACATCCTTCGATAAGACTG \\
GAPDH F & CCAGGTGGTCTCCTCTGA \\
GAPDH R & GCTGTAGCCAAATCGTTGT
\end{tabular}

AQP5, aquaporin 5; MMP, matrix metalloproteinase; F, forward; $\mathrm{R}$, reverse.

Reverse transcription-quantitative PCR (RT-qPCR). Total RNA from eutopic and ectopic ESCs was extracted using TRIzol ${ }^{\circledR}$ reagent (cat. no. 15596018; Invitrogen; Thermo Fisher Scientific, Inc.). Total RNA (1 $\mu \mathrm{g})$ was reverse transcribed to cDNA using PrimeScript ${ }^{\mathrm{TM}}$ RT Master Mix (cat. no. RR036B; Takara Biotechnology Co., Ltd.) at $42^{\circ} \mathrm{C}$ for $1 \mathrm{~h}$. RT-qPCR was performed in a 7300 real-time PCR system (Applied Biosystems; Thermo Fisher Scientific, Inc.) using TB Green ${ }^{\circledR}$ Premix Ex Taq ${ }^{\mathrm{TM}}$ II (cat. no. RR820Q; Takara Biotechnology Co., Ltd.) under the following conditions: Incubation at $95^{\circ} \mathrm{C}$ for $30 \mathrm{~min}$, followed by amplification at $95^{\circ} \mathrm{C}$ for $35 \mathrm{sec}, 60^{\circ} \mathrm{C}$ for $34 \mathrm{sec}$ and $72^{\circ} \mathrm{C}$ for $35 \mathrm{sec}$, for a total of 40 cycles, and a final extension step at $72^{\circ} \mathrm{C}$ for $5 \mathrm{~min}$. The relative expression levels were calculated using the $2^{-\triangle \Delta C q}$ method (17) and normalized to the expression levels of GAPDH. The sequences of primers (provided by Sangon Biotech Co., Ltd.) used are shown in Table I.

CCK- 8 assay. The cells (5x104/well) were plated in 96-well plates. After siRNA transfection for $24 \mathrm{~h}$, cell viability was determined using the CCK-8 kit (cat. no. CA1210; Beijing Solarbio Science \& Technology Co., Ltd.). After siRNA transfection, the cells were cultivated for 24,48 and $72 \mathrm{~h}$. Subsequently, CCK-8 solution (10 $\mu \mathrm{l})$ was added to the 96-well plates, and the cells were cultured for another $4 \mathrm{~h}$. The absorbance was then determined at $450 \mathrm{~nm}$ using a microplate reader (SpectraMax iD5; Molecular Devices, LLC).

Scratch wound-healing assay. The cells (5x10 $/$ well) were cultivated to $80-90 \%$ confluence in 6-well plates. After the medium had been discarded, confluent cells were scratched using a $10-\mu 1$ pipette tip, washed with serum-free DMEM and incubated at $37^{\circ} \mathrm{C}$ for $48 \mathrm{~h}$. Migration was evaluated by counting migrating cells from five random fields under a 100x inverted microscope (Ts2r-FL; Nikon Corporation). The following formula [(1-the distance following healing/the 
Table II. List of primary antibodies used for western blotting.

\begin{tabular}{lll}
\hline Target & \multicolumn{1}{c}{ Antibody name } & Cat. no. \\
\hline AQP2 & Rabbit Anti-Aquaporin 2 antibody (EPR21080) & ab199975 \\
AQP5 & Rabbit Anti-Aquaporin 5 antibody & ab78486 \\
AQP8 & Rabbit Anti-AQP8 antibody (EPR8397) & ab133667 \\
MMP-2 & Rabbit Anti-MMP2 antibody (EPR17003-25) & ab181286 \\
MMP-9 & Rabbit Anti-MMP9 antibody & ab73734 \\
E-cadherin & Rabbit Anti-E Cadherin antibody (EP700Y) & ab40772 \\
N-cadherin & Rabbit Anti-N Cadherin antibody & ab18203 \\
GAPDH & Mouse Anti-GAPDH antibody (6C5)-Loading Control & ab8245
\end{tabular}

$\mathrm{AQP}$, aquaporin; MMP, matrix metalloproteinase.

distance prior to healing) x100] was used to calculated relative migration rate of cells.

Transwell invasion assay. Transwell chambers (8- $\mu \mathrm{m}$ pores; Corning Inc.) coated in Matrigel (BD Biosciences) were used to determine the invasiveness of cells. The cells $\left(1 \times 10^{5} /\right.$ well in a 24-well plate) were inserted into the upper chambers of Transwell chambers, which contained $200 \mu$ DMEM, whereas $500 \mu \mathrm{l}$ DMEM containing 10\% FBS was added into the lower chamber as the chemoattractant. The cells were incubated at $37^{\circ} \mathrm{C}$ for $48 \mathrm{~h}$. Subsequently, the cells adhered to the upper surface were removed using a cotton swab, whereas those on the lower surface were fixed with $4 \%$ pre-cold methanol for $15 \mathrm{~min}$, and stained with $0.1 \%$ crystal violet solution (Sigma-Aldrich; Merck KGaA) for $20 \mathrm{~min}$ at room temperature. Cells were counted from a minimum of 10 fields per filter under a 100x light microscope (Ts2r-FL; Nikon Corporation).

Western blotting. Tissue and cell lysates of eutopic and ectopic endometrium samples were lysed for protein isolation and analyzed as previously described (18). Total proteins were extracted using RIPA buffer (cat. no. R0010; Beijing Solarbio Science \& Technology Co., Ltd.). The supernatant from total proteins was collected and the protein concentration was determined using the BCA protein assay kit (cat. no. PC0020; Beijing Solarbio Science \& Technology Co., Ltd.). The proteins (30 $\mu \mathrm{g}$ /lane) were separated by SDS-PAGE on $12 \%$ gels and transferred onto PVDF membranes (cat. no. IPVH00010; EMD Millipore) using electroblotting apparatus. The membranes were then blocked with $5 \%(\mathrm{w} / \mathrm{v})$ skimmed milk in Tris-buffered saline containing $0.5 \%$ Tween-20 (w/v) buffer for $1 \mathrm{~h}$ at $37^{\circ} \mathrm{C}$. The membranes were probed with specific primary antibodies (Abcam), as listed in Table II, at $4^{\circ} \mathrm{C}$ overnight, and then washed with TBS containing $0.1 \%$ Tween-20. The membranes were then incubated with secondary antibodies [horseradish peroxidase (HRP)-conjugated secondary antibody (goat anti-mouse; cat. no. ab205719; Abcam) and HRP-conjugated secondary antibody (Goat Anti-Rabbit; cat. no. ab205718; Abcam)] at a dilution of 1:2,000 for $1 \mathrm{~h}$ at $37^{\circ} \mathrm{C}$. After washing the membranes three times at an interval of $10 \mathrm{~min}$, the signals were visualized using an ECL detection kit (Promega Corporation) and normalized to GAPDH. Bio-Rad ChemiDoc ${ }^{\mathrm{TM}}$ XRS+ system with Image $\mathrm{Lab}^{\mathrm{TM}}$
Software version 4.1 (Bio-Rad Laboratories, Inc.) were used to semi-quantify band intensities.

Statistical analysis. Data are presented as the mean \pm standard deviation. Statistical significance was analyzed by one-way ANOVA followed by Dunnett's post hoc test to compare the differences. $\mathrm{P}<0.05$ was considered to indicate a statistically significant difference. The analyses were performed using SPSS 17.0 software (SPSS, Inc.).

\section{Results}

Observation of pathological features in AM. The normal endometrium, deep muscular, superficial muscular and subserous layers of the pathological sections of the mice are shown in Fig. 1A. Notably, ESCs and glands infiltrated the deep muscular, superficial muscular and subserous layers of the pathological sections of the mice in AM groups. It was revealed that AM severity was higher in the AM-I and AM-II groups compared with the sham group (Fig. 1B; $\mathrm{P}<0.001$ ), which indicated that the model was successfully established. Similarly, uterus diameter was longer (Fig. $1 C$; $P<0.01$ ), uterus index was higher (Fig. 1D; $\mathrm{P}<0.001)$ and there were a greater number of nodules on the uterine surface (Fig. 1F; $\mathrm{P}<0.001)$ in the AM-I and AM-II groups compared with the sham treatment group. However, no statistically significant differences were found in ovary index among the control, sham, AM-I or AM-II groups, which may indicate that that there was no effect on the ovaries (Fig. 1E; P>0.05). These data suggested that the AM model was successfully constructed and could be used in subsequent experiments.

AQP2, AQP5 and AQP8 are highly-expressed in eutopic and ectopic endometrial tissues from AM mice, particularly in ectopic endometrium. AQP2 (Fig. 2A and $\mathrm{B} ; \mathrm{P}<0.001$ ), AQP5 (Fig. 2A and $\mathrm{C}$; $\mathrm{P}<0.001$ ) and AQP8 (Fig. 2A and D; $\mathrm{P}<0.001)$ were highly expressed in the eutopic endometrium of AM-I- and AM-II-treated mice compared with the sham treatment group. In addition, the expression levels of AQP2 (Fig. 2E and F), AQP5 (Fig. 2E and G) and AQP8 (Fig. 2E and $\mathrm{H}$ ) were highly expressed in the ectopic endometrium of AM-I and AM-II mice compared with the sham treatment group. 


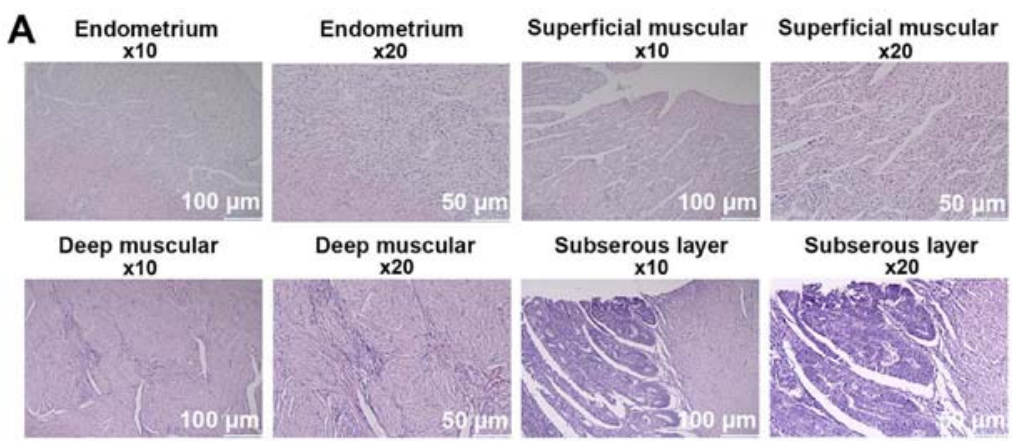

B

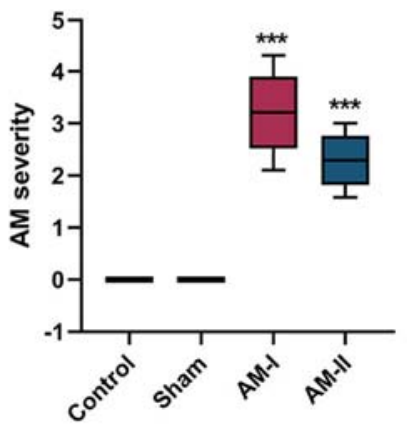

D

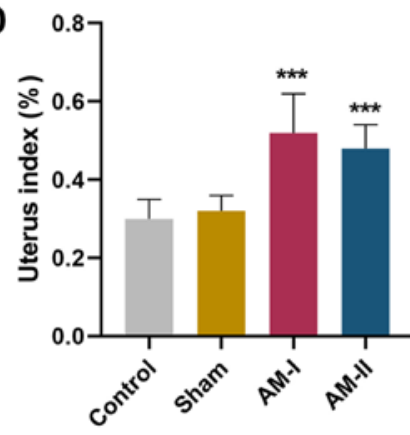

C

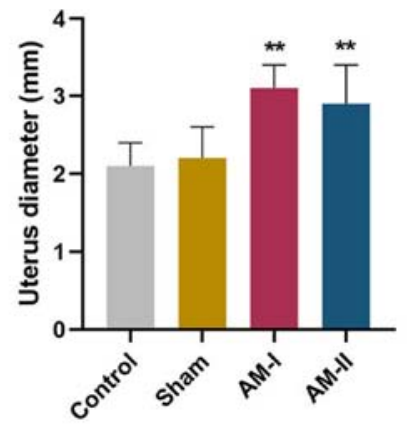

$\mathbf{E}$

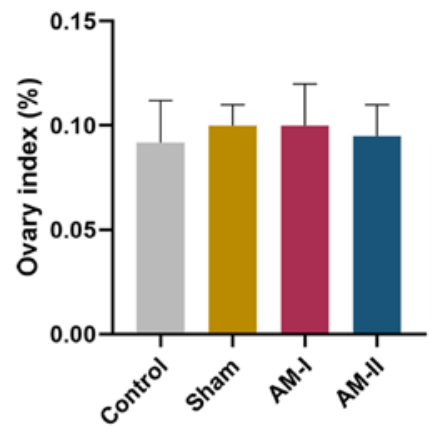

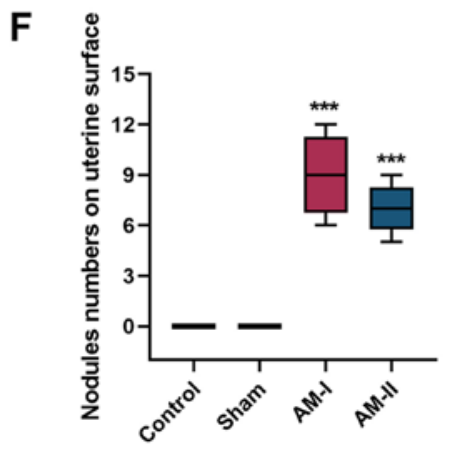

Figure 1. Observation and grading of organizational morphology. (A) Hematoxylin-eosin was used to assess the pathological features of AM (magnification, x10 or x20). (B) Degree of AM, (C) uterus diameter, (D) uterus index, (E) ovary index and (F) number of nodules on the uterine surface of control, sham, AM-I and AM-II groups. Data are presented as the mean $\pm \mathrm{SD}$. ${ }^{* *} \mathrm{P}<0.01$ or ${ }^{* * *} \mathrm{P}<0.001$ vs. sham. AM, adenomyosis.

siAQP5 suppresses the viability, migration, invasion and EMT of ESCs. As shown in Fig. 3A, vimentin was positively expressed in cells from eutopic and ectopic endometrium, which according to previous research (19) indicated that these cells were ESCs (purity $>99 \%$ ). To investigate if low expression levels of AQP5 can alleviate AM, the effects of knockdown of AQP5 expression were examined on cell viability, migration, invasion and EMT in the cells obtained from eutopic and ectopic endometrium. As shown in Fig. 3B and C, the mRNA expression levels of AQP5 were significantly reduced in eutopic and ectopic ESCs following transfection with siAQP5
$(\mathrm{P}<0.001)$. In addition, as shown in Fig. 3D and E, compared with the control, reduced expression of AQP5 suppressed viability of eutopic and ectopic ESCs at $48 \mathrm{~h}(\mathrm{P}<0.05)$ and $72 \mathrm{~h}$ $(\mathrm{P}<0.001)$. The effects of siAQP5 on migration and invasion of eutopic and ectopic ESCs were evaluated by performing scratch wound-healing assays and Transwell assays, respectively. Compared with the blank control group, siAQP5 significantly suppressed the migration of eutopic and ectopic ESCs (Fig. 4A and B; P<0.001). Similarly, compared with the blank control group, siAQP5 significantly suppressed the invasion of eutopic and ectopic ESCs (Fig. 4C and D; P<0.001). As 
A

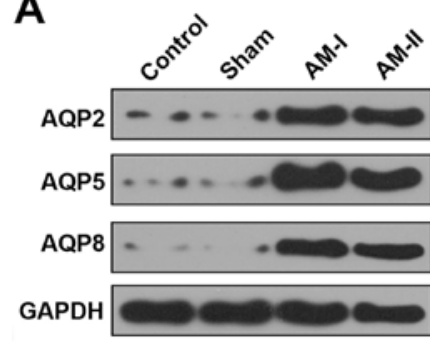

C

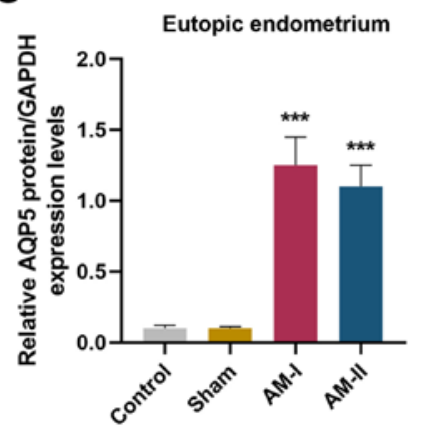

E

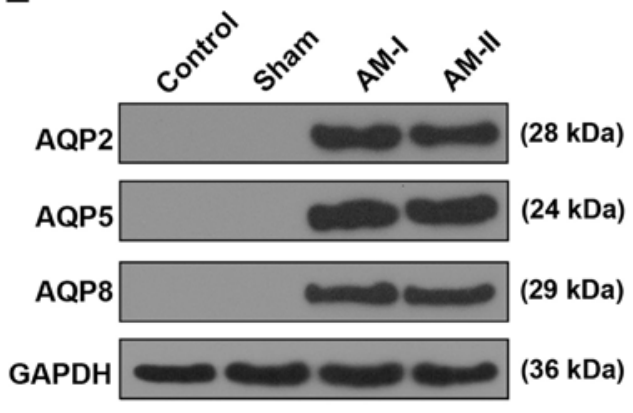

G

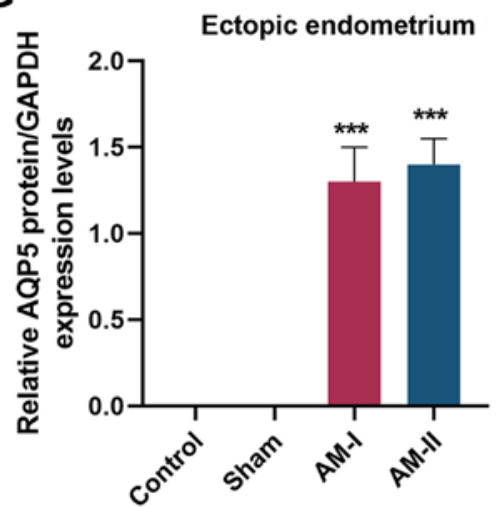

B

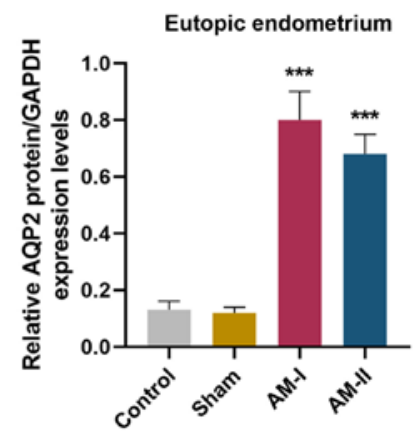

D

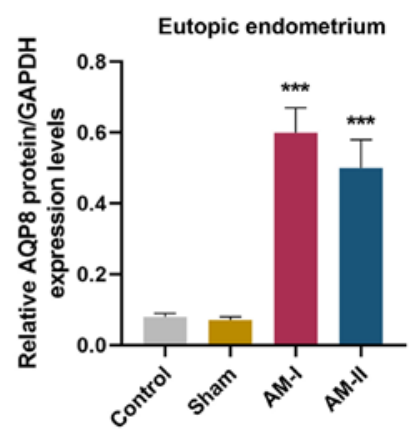

$\mathbf{F}$

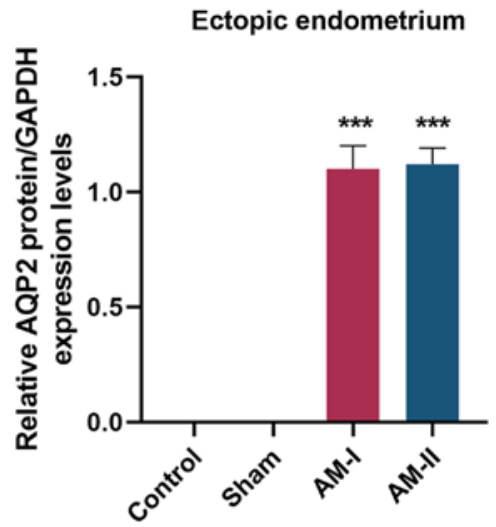

H

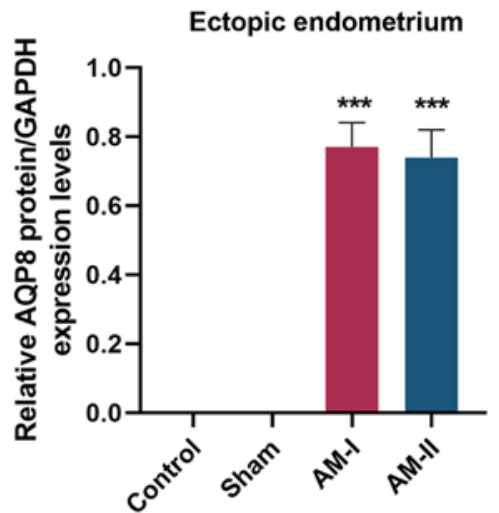

Figure 2. Expression levels of AQP2, AQP5 and AQP8 in eutopic and ectopic endometrial samples were detected by western blot analysis. (A) Western blot analysis of AQP2, AQP5 and AQP8 from the eutopic endometrium. Protein expression levels of (B) AQP2, (C) AQP5 and (D) AQP8 in the eutopic endometrium of control, sham, AM-I and AM-II groups were semi-quantified. (E) Western blot analysis of AQP2, AQP5 and AQP8 from ectopic endometrium. Protein expression levels of (F) AQP2, (G) AQP5 and (H) AQP8 in the ectopic endometrium of control, sham, AM-I and AM-II groups were semi-quantified. Data are presented as the mean $\pm \mathrm{SD} .{ }^{* * * *} \mathrm{P}<0.001$ vs. sham. $\mathrm{AM}$, adenomyosis; $\mathrm{AQP}$, aquaporin.

shown in Fig. 5A-F, compared with the control group, siAQP5 markedly reduced the mRNA and protein expression levels of MMP-2, MMP-9 and N-cadherin, but increased the mRNA and protein expression levels of E-cadherin in eutopic and ectopic ESCs $(\mathrm{P}<0.001)$.

\section{Discussion}

Cell attachment, adhesion and invasion are important in maintaining the normal functions of the endometrium, but it is believed that these properties may participate in the 

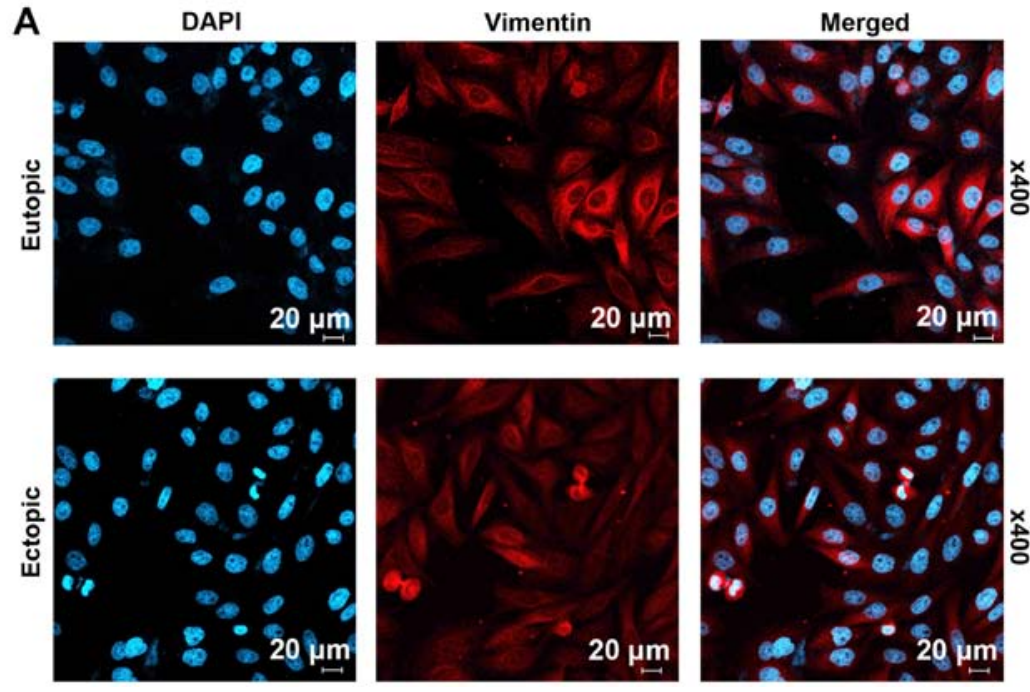

B

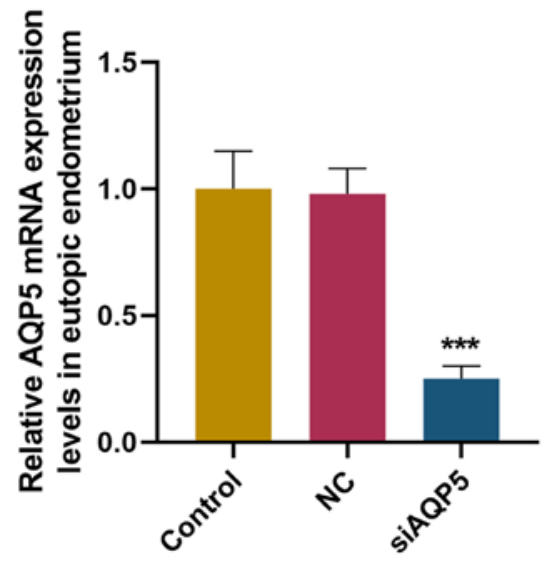

D

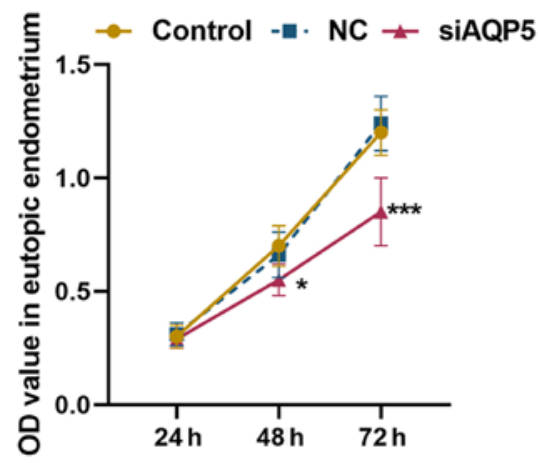

C

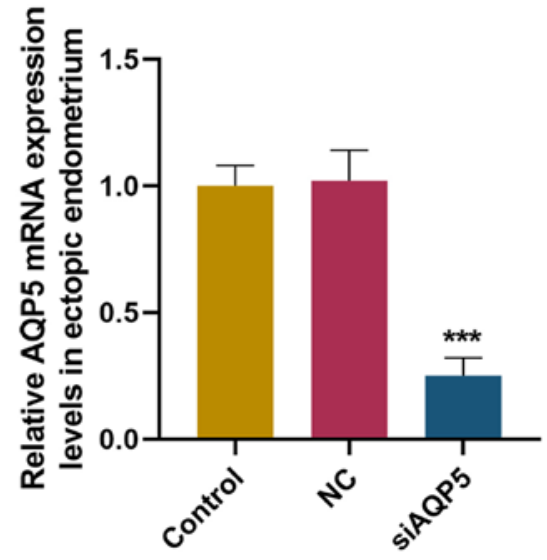

E

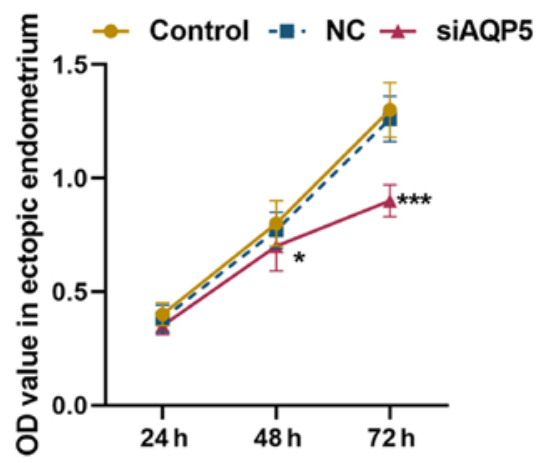

Figure 3. Identification of human eutopic and ectopic ESCs, and the effects of siAQP5 on the viability of eutopic and ectopic ESCs. (A) ESCs in eutopic and ectopic endometrium were identified by immunofluorescence. mRNA expression levels of AQP5 in (B) eutopic and (C) ectopic ESCs from the control, NC and siAQP5 groups were detected by reverse transcription-quantitative PCR. Viability of (D) eutopic and ectopic ESCs from the control. (E) Before ectopic ESCs., $\mathrm{NC}$ and siAQP5 groups was detected by Cell Counting kit-8 assay. Data are presented as the mean $\pm \mathrm{SD}$. ${ }^{*}<0.05$ or ${ }^{* * *} \mathrm{P}<0.001$ vs. control. AQP, aquaporin; NC, negative control; siAQP5, small interfering RNA targeting AQP5; ESCs, endometrial stromal cells.

development of endometriosis (20). In addition, adhesion, invasion and growth of the endometrium towards the muscle layer to form ectopic endometrium is seen as a key event in the pathogenesis of AM (21). Thus, understanding the mechanisms underlying cell viability, migration and invasion of the endometrium may help understand and potentially help develop a future treatment for AM.
The present study revealed that AM severity and uterus index were higher, uterus diameter was longer and there were a greater number of nodules on the uterine surface in the AM model of female mice compared with in the control mice. This suggested that estrus could regulate the severity of AM in female mice. In addition, AQP2, AQP5 and AQP8 were highly expressed in the eutopic and ectopic endometrium of 
A
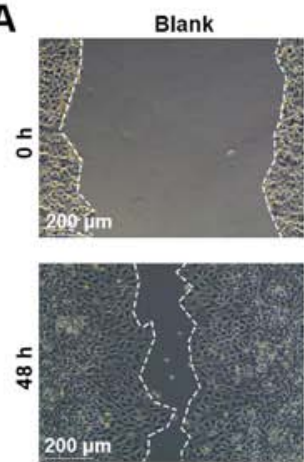

B

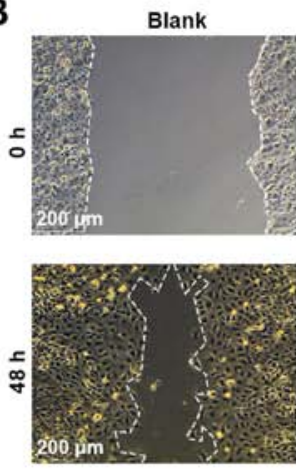

C
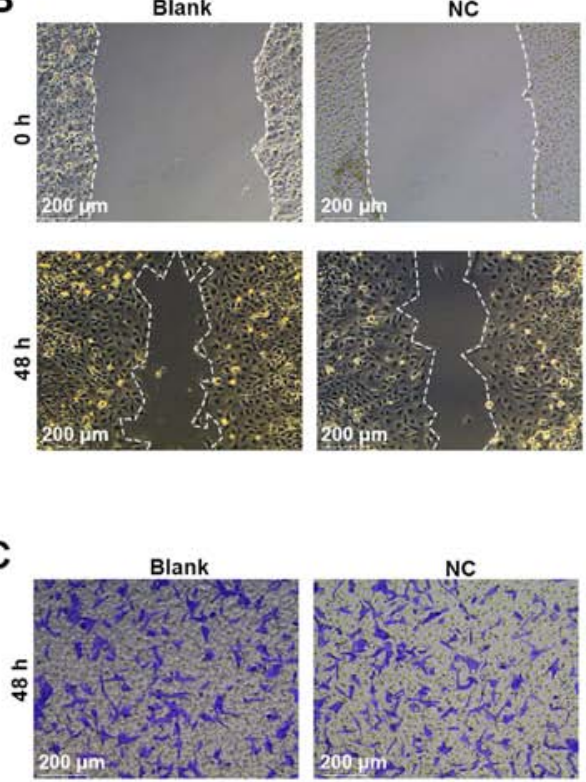
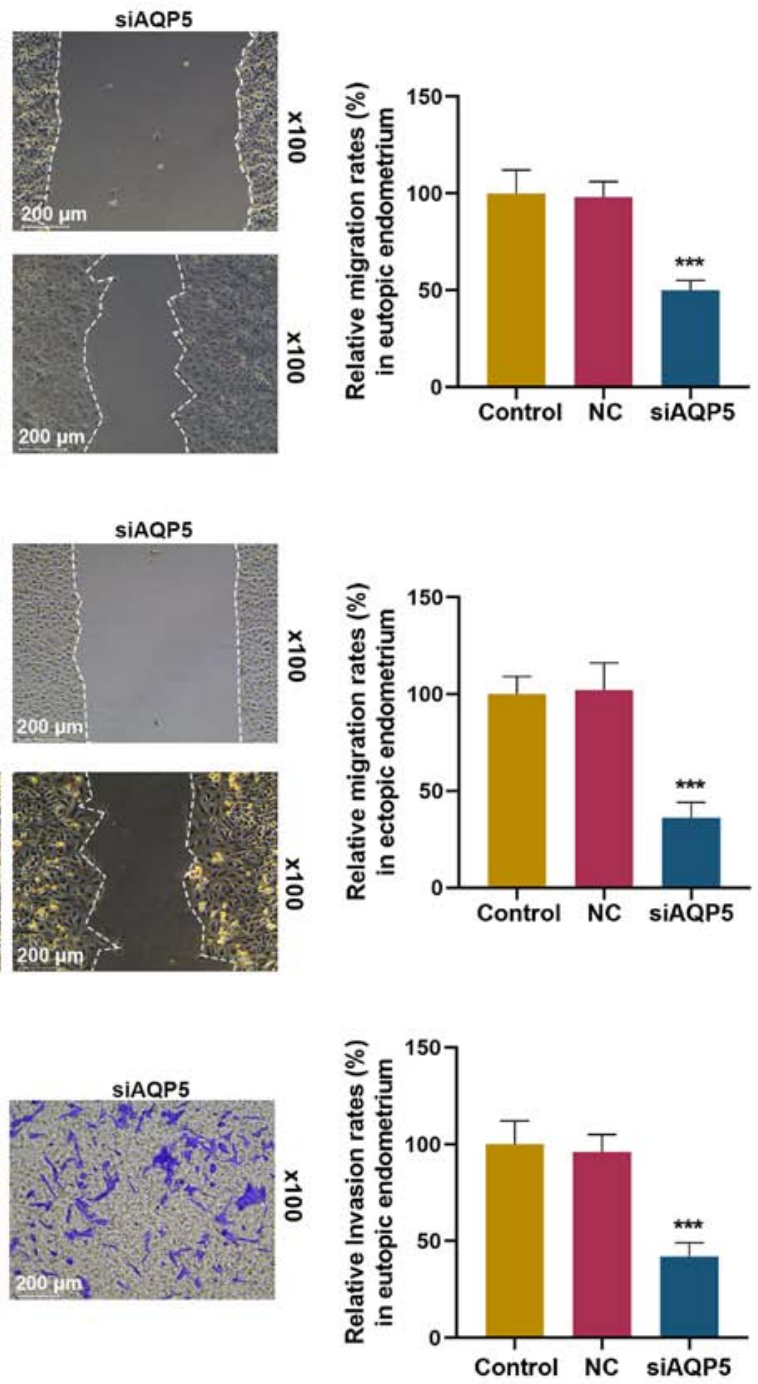

D
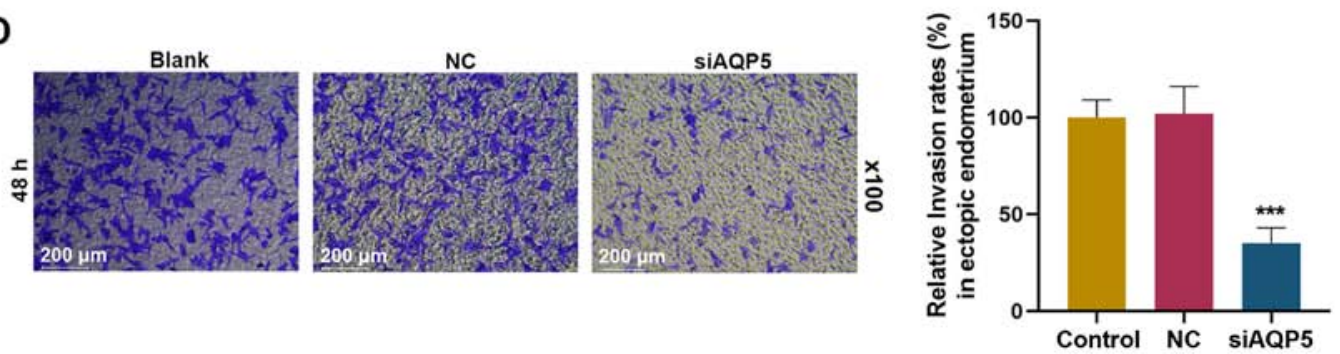

Figure 4. Knockdown of AQP5 suppressed cell migration and invasion of eutopic and ectopic ESCs. Cell migration of (A) eutopic and (B) ectopic ESCs from the control, NC and siAQP5 groups was detected by scratch wound-healing assay. Cell invasion of (C) eutopic and (D) ectopic ESCs from the control, NC and siAQP5 groups was detected by Transwell invasion assay. Data are presented as the mean $\pm \mathrm{SD}$. ${ }^{* * *} \mathrm{P}<0.001$ vs. control. AQP, aquaporin; NC, negative control; siAQP5, small interfering RNA targeting AQP5; ESCs, endometrial stromal cells.

AM-I and AM-II model mice. Therefore, the role of AQP5 in ESCs extracted from the eutopic and ectopic endometrium of patients with AM was further investigated. The results indicated that AQP5 silencing suppressed the viability of ESCs from the eutopic and ectopic endometrium. In addition, it was found that AQP5 silencing inhibited the migration of ESCs, similar to a previous study which reported that AQP5 silencing inhibited proliferation and migration of ectopic endometrial glandular epithelial cells in endometriosis (13). It has also previously been suggested that AQP5 may affect cell proliferation and migration during tumor development. For example, downregulating AQP5 expression inhibited the proliferation and migration of human gastric carcinoma cells (22), human glioma cells (23) and prostate cancer cells (24). Downregulating AQP5 in Ishikawa cells has also been shown to reduce cell migration (25).

The current study found that AQP5 silencing inhibited cell viability, migration and invasion, which aligns with a previous study in human non-small cell lung cancer, which demonstrated that AQP5 expression promoted cell invasion (26). In 
A

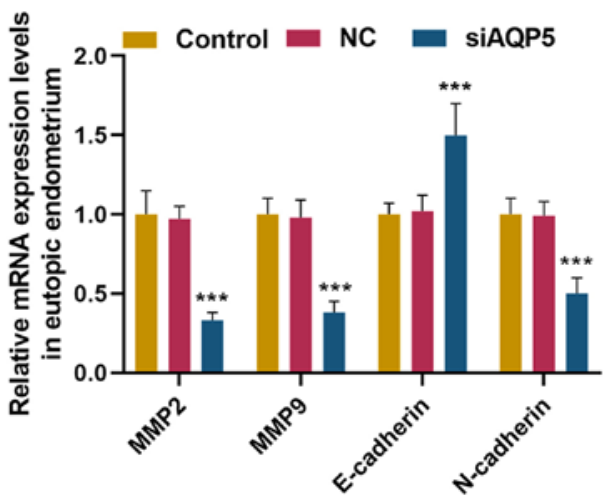

C

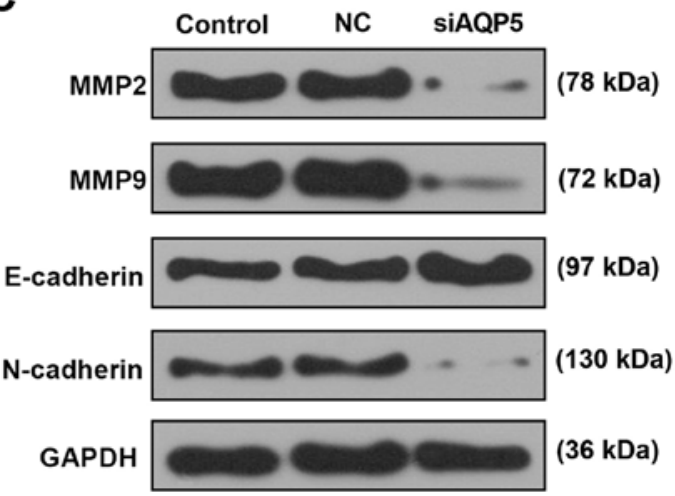

E

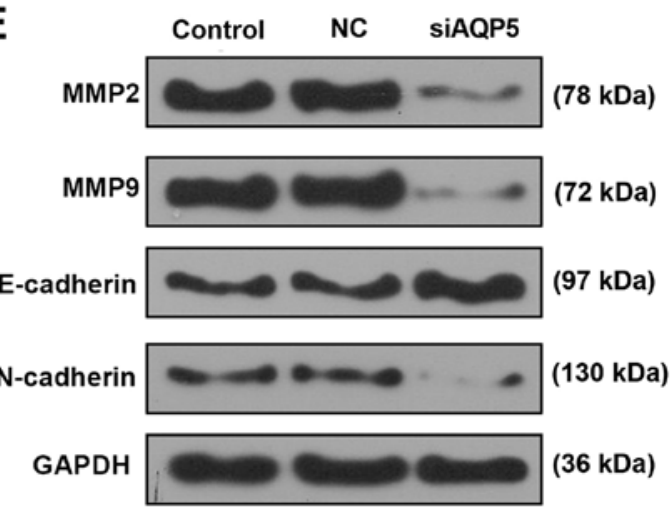

B

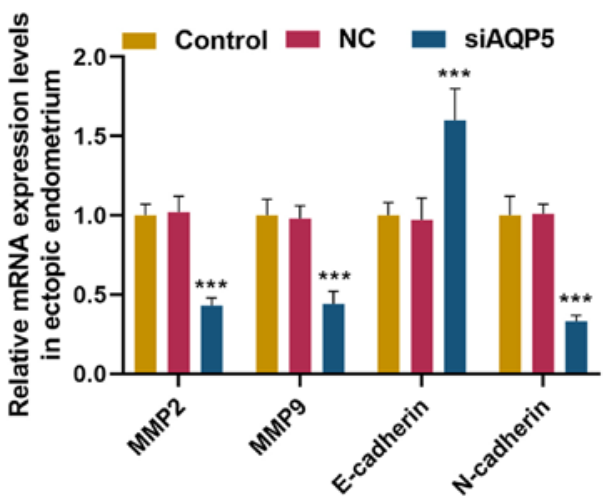

D

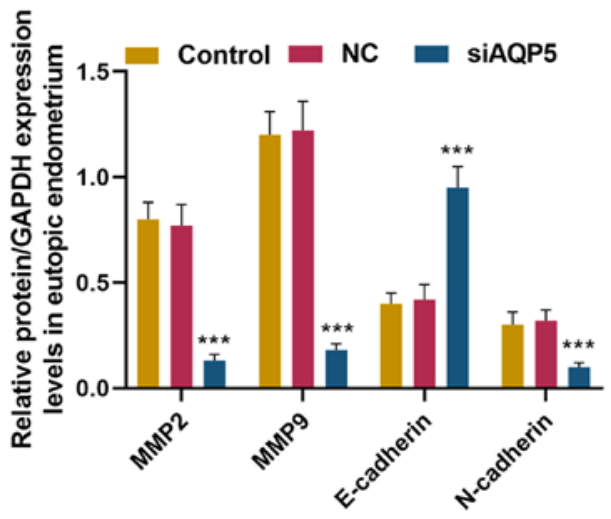

$\mathbf{F}$

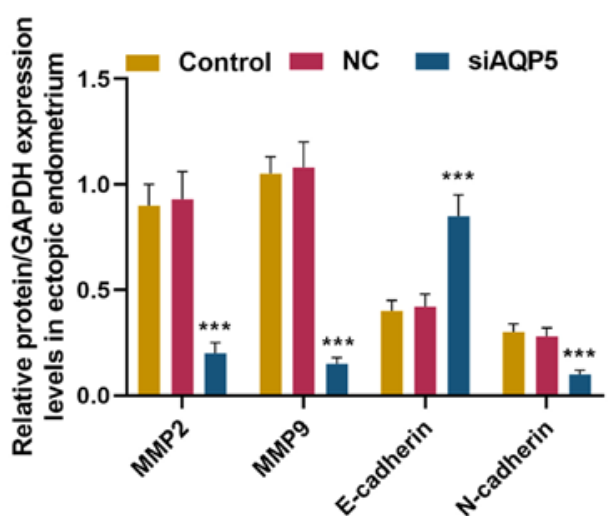

Figure 5. Knockdown of AQP5 suppressed epithelial-mesenchymal transition, and MMP-2 and MMP-9 expression in eutopic and ectopic ESCs mRNA expression levels of E-cadherin, N-cadherin, MMP-2 and MMP-9 from (A) eutopic and (B) ectopic ESCs from the control, NC and siAQP5 groups were detected by reverse transcription-quantitative PCR. Protein expression levels of E-cadherin, N-cadherin, MMP-2 and MMP-9 in the (C and D) eutopic and (E and F) ectopic ESCs of the control, NC and siAQP5 groups were detected by western blotting. Data are presented as the mean $\pm \mathrm{SD}$. ${ }^{* * *} \mathrm{P}<0.001 \mathrm{vs}$. control. AQP, aquaporin; MMP, matrix metalloproteinase; NC, negative control; siAQP5, small interfering RNA targeting AQP5; ESCs, endometrial stromal cells.

addition, the mRNA and protein expression levels of MMP-2 and MMP-9, and some EMT-related genes (N-cadherin and E-cadherin) were determined; the results revealed that AQP5 silencing inhibited the mRNA and protein expression levels of MMP-2, MMP-9 and N-cadherin, but promoted E-cadherin of ESCs. MMPs are a family of zinc-dependent proteolytic enzymes capable of degradation and reconstruction of the extracellular matrix, which can lead to cell migration and invasion (27). MMP-2 and MMP-9 degrade type IV collagen and gelatin substrates (28). MMP-2 and MMP-9 expression levels have been reported to be higher in the eutopic and ectopic endometrial tissues of patients with AM compared with healthy tissues (29). Thus, increased MMP-2 and MMP-9 expression may play an important role in the development of AM, potentially through increasing the invasiveness of endometrial tissues into the myometrium (30). EMT transforms epithelial cells into mesenchymal cells (31). During EMT, loss of cell-cell adhesion is related to the migratory and invasive characteristics of cells (32). EMT is characterized by decreased E-cadherin and corresponding increased N-cadherin expression (33). Therefore, the current data suggested that AQP5 silencing inhibited the invasion and migration of cells, which 
may be caused by the regulation of EMT, and MMP-2 and MMP-9 expression. A similar report revealed that downregulation of AQP5 inhibited hepatocellular carcinoma cell invasion and EMT process through modulating EMT-related molecules, such as E-cadherin and N-cadherin (34). Wang et al (35) reported that AQP5 silencing inhibited proliferation, migration and the enzyme activity of MMP-9 in human umbilical vein endothelial cells, but did not affect the enzyme activity of MMP-2. The discrepancy between this previous report and the current study with regards to MMP-2 may have resulted from different detection indexes (i.e. enzyme activity and protein expression). Collectively, silencing of AQP5 expression and/or function might be a potential useful treatment to prevent the development of AM; however, the method by which AQP5 could be silenced requires further study.

This study indicated that the effects of AQP5 on AM may be fundamental for the development of novel therapeutic targets, or reliable diagnostic and prognostic biomarkers. However, the pathological images were not high quality. In addition, cytokeratin, von Willebrand factor and $\alpha$-smooth muscle actin could be applied to identify ESCs, which were not applied in this study. Further research is required to identify the underlying mechanisms and signaling pathways of AQP5 on AM, and such studies will reveal whether AQP5 and downstream effectors could be potential targets for developing new therapeutics for AM.

Taken together, these findings revealed that AQP5 was highly expressed in eutopic and ectopic endometrial samples in a mouse model of AM. In addition, AQP5 silencing suppressed viability, migration, invasion, EMT, and MMP-2 and MMP-9 protein expression in ESCs derived from patients with AM. These results may provide a new basis for the development of future therapeutics for AM through the inhibition of the expression and function of AQP channels. The effects of depleting AQP2 and AQP8, along with the pathogenic role of these transmembrane channels in malignancy, AM and endometriosis should also be further explored.

\section{Acknowledgements}

Not applicable.

\section{Funding}

The current study was supported by the Medical Scientific Research Project of Hainan Province (grant no. 18A200093).

\section{Availability of data and materials}

The datasets used and/or analyzed during the current study are available from the corresponding author on reasonable request.

\section{Authors' contributions}

LJ and YL developed the concept and designed the study. YH and $\mathrm{XZ}$ conducted data acquisition, analysis and interpretation, LJ, YL and FW conducted the majority of experiments, drafted the manuscript and critically revised it for important intellectual content. All authors read and approved the final manuscript.

\section{Ethics approval and consent to participate}

The current study was approved by the Ethics Committee of the Second Affiliated Hospital of Hainan Medical University (SA20180111022). Written informed consent was obtained from all patients. All animal experiments were approved by the Institutional Committee for Animal Research of The Second Affiliated Hospital of Hainan Medical University and in accordance with National Guidelines for the Care and Use of Laboratory Animals.

\section{Patient consent for publication}

Not applicable.

\section{Competing interests}

The authors declare that they have no competing interests.

\section{References}

1. Struble J, Reid S and Bedaiwy MA: Adenomyosis: A clinical review of a challenging gynecologic condition. J Minim Invasive Gynecol 23: 164-185, 2016.

2. Harada T, Khine YM, Kaponis A, Nikellis T, Decavalas G and Taniguchi F: The lity. Obstet Gynecol Surv 71: 557-568, 2016.

3. Vlahos NF, Theodoridis TD and Partsinevelos GA: Myomas and adenomyosis: Impact on reproductive outcome. Biomed Res Int 2017: 5926470, 2017

4. Pontis A, D'Alterio MN, Pirarba S, de Angelis C, Tinelli R and Angioni S: Adenomyosis: A systematic review of medical treatment. Gynecol Endocrinol 32: 696-700, 2016.

5. Li C and Wang W: Molecular biology of aquaporins. Adv Exp Med Biol 969: 1-34, 2017.

6. Soveral $\mathrm{G}$ and Casini A: Aquaporin modulators: A patent review (2010-2015). Expert Opin Ther Pat 27: 49-62, 2017.

7. Beitz E, Golldack A, Rothert M and von Bulow J: Challenges and achievements in the therapeutic modulation of aquaporin functionality. Pharmacol Ther 155: 22-35, 2015.

8. He Y, Zhou L, Fan Z, Liu S and Fang W: Palmitic acid, but not high-glucose, induced myocardial apoptosis is alleviated by $\mathrm{N}$-acetylcysteine due to attenuated mitochondrial-derived ROS accumulation-induced endoplasmic reticulum stress. Cell Death Dis 9: 568, 2018.

9. García-Solares J, Donnez J, Donnez O and Dolmans MM: Pathogenesis of uterine adenomyosis: Invagination or metaplasia? Fertil Steril 109: 371-379, 2018.

10. Benagiano G, Brosens I and Habiba M: Structural and molecular features of the endomyometrium in endometriosis and adenomyosis. Hum Reprod Update 20: 386-402, 2014.

11. Zhou YF, Mori T, Nagasawa H, Nakayama T, Kubota $T$ and Sakamoto S: Probucol, a hypocholesterolemic agent, prevents the development of uterine adenomyosis induced by pituitary grafting in mice. Anticancer Res 24: 2209-2212, 2004.

12. Jiang XX, Fei XW, Zhao L, Ye XL, Xin LB, Qu Y, Xu KH, Wu RJ and Lin J: Aquaporin 5 plays a role in estrogen-induced ectopic implantation of endometrial stromal cells in endometriosis. PLoS One 10: $\mathrm{e} 0145290,2015$.

13. Xin LB, Jiang XX, Ye XL, Wu RJ, Xu KH, Ma JY and Lin J: AQP5 gene silencing inhibits proliferation and migration of ectopic endometrial glandular epithelial cells in endometriosis. Zhejiang Da Xue Xue Bao Yi Xue 44: 285-292, 2015 (In Chinese).

14. National Research Council Institute for Laboratory Animal Research: In: Guide for the Care and Use of Laboratory Animals. National Academies Press (US) Copyright 1996 by the National Academy of Sciences. All rights reserved., Washington (DC), 1996.

15. Koujyo T, Hatakeyama S, Yamada H, Iwabuchi K, Kajino K, Ogasawara K, Onoe K and Fujimoto S: Induction of endometriosis and adenomyosis by transvaginal pituitary transplantation in mice with and without natural killer cell activity. Am J Reprod Immunol 40: 441-446, 1998. 
16. Mori T, Kyokuwa M and Nagasawa $\mathrm{H}$ : Animal model of uterine adenomyosis: Induction of the lesion in rats by ectopic pituitary isografting. Lab Anim Sci 48: 64-68, 1998.

17. Livak KJ and Schmittgen TD: Analysis of relative gene expression using different real-time quantitative PCR and 2(-Delta Delta C(T)) method. Methods 402-408, 2001.

18. Li Q, Yang T, Li D, Ding F, Bai G, Wang W and Sun H: Knockdown of aquaporin-5 sensitizes colorectal cancer cells to 5 -fluorouracil via inhibition of the Wnt- $\beta$-catenin signaling pathway. Biochem Cell Biol 96: 572-579, 2018

19. Cheong ML, Lai TH and Wu WB: Connective tissue growth factor mediates transforming growth factor $\beta$-induced collagen expression in human endometrial stromal cells. PLoS One 14: e0210765, 2019.

20. Sundqvist J, Andersson KL, Scarselli G, Gemzell-Danielsson K and Lalitkumar PG: Expression of adhesion, attachment and invasion markers in eutopic and ectopic endometrium: A link to the aetiology of endometriosis. Hum Reprod 27: 2737-2746, 2012.

21. Yamin $\mathrm{Hu}$ and Bing $\mathrm{ZH}$ : Expression of VEGF-C and ICAM-1 in adenomyosis and clinical significance. Acta Med Univ Sci Technol Huazhong 38: 217-219, 2009.

22. Huang YH, Zhou XY, Wang HM, Xu H, Chen J and Lv NH: Aquaporin 5 promotes the proliferation and migration of human gastric carcinoma cells. Tumour Biol 34: 1743-1751, 2013.

23. Wang S, Zhao X, Yang S, Chen B and Shi J: Salidroside alleviates high glucose-induced oxidative stress and extracellular matrix accumulation in rat glomerular mesangial cells by the TXNIP-NLRP3 inflammasome pathway. Chem Biol Interact 278 : 48-53, 2017.

24. Li J, Wang Z, Chong T, Chen H, Li H, Li G, Zhai X and Li Y: Over-expression of a poor prognostic marker in prostate cancer: AQP5 promotes cells growth and local invasion. World J Surg Oncol 12: 284, 2014

25. Jiang XX, Xu KH, Ma JY, Tian YH, Guo XY, Lin J and Wu RJ: Reduced migration of Ishikawa cells associated with downregulation of aquaporin-5. Oncol Lett 4: 257-261, 2012.

26. Chae YK, Woo J, Kim MJ, Kang SK, Kim MS, Lee J, Lee SK Gong G, Kim YH, Soria JC, et al: Expression of aquaporin 5 (AQP5) promotes tumor invasion in human non small cell lung cancer. PLoS One 3: e2162, 2008.
27. Chen Q, Jin M, Yang F, Zhu J, Xiao Q and Zhang L: Matrix metalloproteinases: Inflammatory regulators of cell behaviors in vascular formation and remodeling. Mediators Inflamm 2013: 928315, 2013.

28. Webb AH, Gao BT, Goldsmith ZK, Irvine AS, Saleh N, Lee RP, Lendermon JB, Bheemreddy R, Zhang Q, Brennan RC, et al: Inhibition of MMP-2 and MMP-9 decreases cellular migration, and angiogenesis in in vitro models of retinoblastoma. BMC Cancer 17: 434, 2017.

29. Yi KW, Kim SH, Ihm HJ, Oh YS, Chae HD, Kim CH and Kang BM: Increased expression of p21-activated kinase 4 in adenomyosis and its regulation of matrix metalloproteinase-2 and -9 in endometrial cells. Fertil Steril 103: 1089-1097.e2, 2015.

30. Li T, Li YG and Pu DM: Matrix metalloproteinase-2 and -9 expression correlated with angiogenesis in human adenomyosis. Gynecol Obstet Invest 62: 229-235, 2006.

31. Tulchinsky E, Demidov O, Kriajevska M, Barlev NA and Imyanitov E: EMT: A mechanism for escape from EGFR-targeted therapy in lung cancer. Biochim Biophys Acta Rev Cancer 1871: 29-39, 2019.

32. Jakobsen KR, Demuth C, Sorensen BS and Nielsen AL: The role of epithelial to mesenchymal transition in resistance to epidermal growth factor receptor tyrosine kinase inhibitors in non-small cell lung cancer. Transl Lung Cancer Res 5: 172-182, 2016.

33. Ramamurthy VP, Ramalingam S, Gediya LK and Njar VCO: The retinamide VNLG-152 inhibits f-AR/AR-V7 and MNK-eIF4E signaling pathways to suppress EMT and castration-resistant prostate cancer xenograft growth. FEBS J 285: 1051-1063, 2018.

34. He Z, Dong W, Hu J and Ren X: AQP5 promotes hepatocellular carcinoma metastasis via NF- $\kappa$ B-regulated epithelial-mesenchymal transition. Biochem Biophys Res Commun 490: 343-348, 2017.

35. Wang W, Li Q, Yang T, Li D, Ding F, Sun H and Bai G: RNA interference-mediated silencing of aquaporin (AQP)-5 hinders angiogenesis of colorectal tumor by suppressing the production of vascular endothelial growth factor. Neoplasma 65: 55-65, 2018.

This work is licensed under a Creative Commons Attribution-NonCommercial-NoDerivatives 4.0 International (CC BY-NC-ND 4.0) License. 